\title{
AN-Aided Secure Beamforming in SWIPT-Aware Mobile Edge Computing Systems with Cognitive Radio
}

\author{
Zhe Wang, ${ }^{1,2}$ Taoshen $\mathrm{Li}^{1}{ }^{1}$ Jin $\mathrm{Ye} \mathbb{D}^{\circ}{ }^{3}$ Xi Yang $\mathbb{D}^{4,5}$ and Ke Xiong ${ }^{6}$ \\ ${ }^{1}$ School of Computer, Electronics and Information, Guangxi University, Nanning, Guangxi 530004, China \\ ${ }^{2}$ Institute of Artificial Intelligence, Guangxi University for Nationalities, Nanning, Guangxi 530006, China \\ ${ }^{3}$ Guangxi Key Laboratory of Multimedia Communication \& Network Technology, Nanning, Guangxi 530004, China \\ ${ }^{4}$ School of Information, Beijing Wuzi University, Beijing 101149, China \\ ${ }^{5}$ Beijing Intelligent Logistics System Collaborative Innovation Center, Beijing 101149, China \\ ${ }^{6}$ School of Computer and Information Technology, Beijing Jiaotong University, Beijing 100044, China
}

Correspondence should be addressed to Jin Ye; yejin@gxu.edu.cn and Xi Yang; yxyoung@hotmail.com

Received 8 March 2020; Revised 15 May 2020; Accepted 1 July 2020; Published 4 November 2020

Academic Editor: Lisheng Fan

Copyright (c) 2020 Zhe Wang et al. This is an open access article distributed under the Creative Commons Attribution License, which permits unrestricted use, distribution, and reproduction in any medium, provided the original work is properly cited.

\begin{abstract}
Simultaneous wireless information and power transfer (SWIPT) becomes more and more popular in cognitive radio (CR) networks, as it can increase the resource reuse rate of the system and extend the user's lifetime. Due to the deployment of energy harvesting nodes, traditional secure beamforming designs are not suitable for SWIPT-enabled CR networks as the power control and energy allocation should be considered. To address this problem, a dedicated green edge power grid is built to realize energy sharing between the primary base stations (PBSs) and cognitive base stations (CBSs) in SWIPT-enabled mobile edge computing (MEC) systems with CR. The energy and computing resource optimal allocation problem is formulated under the constraints of security, energy harvesting, power transfer, and tolerable interference. As the problem is nonconvex with probabilistic constraints, approximations based on generalized Bernstein-type inequalities are adopted to transform the problem into solvable forms. Then, a robust and secure artificial noise- (AN-) aided beamforming algorithm is presented to minimize the total transmit power of the CBS. Simulation results demonstrate that the algorithm achieves a close-to-optimal performance. In addition, the robust and secure AN-aided CR based on SWIPT with green energy sharing is shown to require a lower transmit power compared with traditional systems.
\end{abstract}

\section{Introduction}

In future mobile edge computing (MEC) networks, a great number of energy-limited internet of things (IoT) devices will be deployed to collect the data of system status and environment $[1,2]$. Providing wireless power transfer capability is of great importance to relieve energy unbalance and prolong the system lifetime. In practice, radio frequency (RF) signals surrounding the communication systems are more controllable and stable to realize the simultaneous wireless information and power transfer (SWIPT) [3-5] in networks [6], which is a significant way to power the energy-limited edge nodes by transferring wireless energy and information simultaneously with the same waveform.

Cognitive radio (CR) is another meaningful way to utilize a spectrum efficiently for resource reusing in MEC networks. With the spectrum sharing in CR, secondary users (SUs) can coexist with the primary base station (PBS) and primary users (PUs) under the condition that the interference caused by the cognitive base station (CBS) is tolerable to PUs. Recently, many researches are considering about applying SWIPT into the CR network with energy-constrained devices to improve network efficiency and stabilization [7-11]. In [7], a SWIPT-enabled CR network was designed, in which 
the CBS provided SWIPT services to the secondary users, and the worst-case of SU for energy harvesting and tolerable interference power for PU were balanced based on the binary search method. This research can alleviate the double nearfar problem in the energy harvesting network to a certain extent.

In light of the relay needs for network, [9] proposed an SWIPT-enabled CR framework in which the CBSs were integrated with relay capabilities to provide relay services opportunistically for the primary users. The novel framework in [9] was proposed to integrate the SWIPT-enabled CR technology into new scenarios and applications. [10] and [11], respectively, studied the wideband sensing problem under worst-case and max-min fairness case with a SWIPT-based CR framework, in order to achieve higher spectrum and energy utilization in wireless sensor networks.

Inspired by the SWIPT-enabled CR framework, we propose a novel mobile edge computing with SWIPT-enabled CR to realize the optimal allocation of energy and computing resources in this paper. CBSs and PBSs are selected with abundant energy to join the edge network, and a dedicated green edge power grid is built to achieve the energy flow and balance between those edge nodes. At the same time, we should also consider the security issues $[12,13]$ in the SWIPT-based network.

The characteristics of the wireless channel determine that the security rate of its physical layer is limited by channel state information (CSI) [14]. In CR networks, the transmission power of CBS in the secondary network is limited to prevent the primary users from interfering, which further affects the security rate [15]. At the same time, the formulation of a network operation strategy should take the limited energy of energy harvesting nodes (EHNs) into account. In fact, there is no cooperation among SUs, PUs, and EHNs in the CR network, so there are errors in channel estimation and quantification on SWIPT-enabled CR links that cannot be ignored. These errors seriously affect the efficiency of beamforming. Therefore, it is necessary to design a robust secure beamforming scheme for SWIPT-enabled CR MEC.

Aiming at the physical layer security of SWIPT, [16-18], respectively, studied the total transmission power minimization, safety rate maximization, and maximum minimum fairness optimization problem. On the other hand, in the SWIPT-based CR network, since the physical location of EHNs is closer to the transmitter to meet the demand of wireless power density for energy harvesting, it is possible for EHNs act as malicious eavesdroppers disturbing the reception of information receivers. Therefore, in the SWIPT design, a secure rate becomes an essential factor. In [16], a secure beamforming design in MISO SWIPT was proposed where there are multiple information receivers, energy receivers, and eavesdroppers coexisting. Similar to $[16,19]$, investigated a worst-case optimal problem under constraints of transmit power and harvested power in MISO SWIPT. Specifically, the secure and robust transmission strategy in the worst-case energy allocation problem for the MISO channel and multiple input and multiple output (MIMO) channel was also studied in $[20,21]$. [22, 23] extended the strategy for multiple eavesdroppers with multiple antennas. For instance, an artificial noise- (AN-) aided transmit beamforming was designed in [24], which is an efficient way to improve the system secrecy rate efficiently. In [24-26], AN-aided transmission strategies for secret SWIPT were proposed in MISO, single input and single output (SISO), and MIMO channels. In [24], both perfect and imperfect CSI were considered for AN-aided SWIPT design. And with the channel uncertainties, [27] investigates the problem based on the bounded CSI and probabilistic CSI error model, respectively. However, the proposed schemes in [24-27] are difficult to be applied into the SWIPT CR network, because the energy harvesting requirement and the interference of a secondary network have not been taken into account.

This paper designs a SWIPT-enabled CR framework for MEC. By considering the energy allocation, power control, and interference constraints, an efficient and robust AN-aided secure beamforming strategy is designed to achieve better system gains. The main contributions of this paper are as follows.

First, a robust secure beamforming strategy for a CR network powered with SWIPT is proposed. The interference caused by the secondary network and the energy harvesting requirement for EHNs are both considered in our framework. By formulating as an inequality constraint in the system models, the security of the SWIPT-enabled CR network is also taken into account to minimize the transmit power.

Second, since the PBSs in the primary network and the CBSs in the secondary network are powered by green energy, we build a dedicated edge power grid made up of those PBSs and CBSs to share their redundant resources with the others. Therefore, energy balancing and computing offloading can be operated efficiently during these edge base stations in the MEC.

Third, a probabilistic CSI model is designed to characterize the CSI error, which is more suitable for delay-sensitive applications than the bounded CSI model. Subsequently, the optimal beamforming solution is solved by software CVX [28], and a 1-D algorithm is designed to approximate the optimal solution of the system with reasonable computational complexity.

Notations. For a square matrix $\mathbf{A}, \mathbf{A}^{H}, \operatorname{Tr}(\mathbf{A})$, and $\operatorname{Rank}(\mathbf{A})$ denote its Hermitian (conjugate) transpose, trace, and rank, respectively. $\mathbb{C}^{N \times M}, \mathbb{R}^{N \times M}$, and $\mathbb{H}^{N}$ denote the set of all the $N \times M$ complex matrices, $N \times M$ real matrices, and $N \times N$ Hermitian matrices. $\mathbf{A}>0(\mathbf{A} \geq 0)$ indicates that matrix $\mathbf{A}$ is positive definite or semidefinite. In particular, the distribution of complex Gaussian random vectors with mean $\mu$ and variance $\sigma^{2}$ is denoted by $\left(\mu, \sigma^{2}\right)$.

\section{System Model}

The SWIPT-enabled CR MEC network is shown in Figure 1, in which the PBS and CBS act as MEC servers for users as well as the CBS providing SWIPT services for the cognitive network. There are $K$ SUs, $I$ PUs, and $M$ ERs coexisting in the network. The CBS is equipped with $N_{t}\left(N_{t}>1\right)$ antennas as the PBS has only one. The SUs and ERs are also equipped with single antenna nodes to harvest RF energy from the 


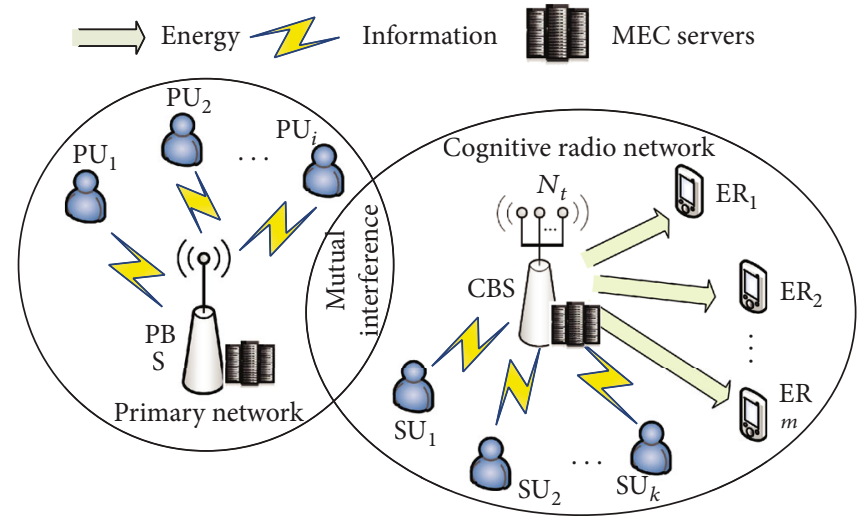

Figure 1: System model.

ambient environment. In practice, the idle users in the cognitive radio network may act as energy receivers (ERs) harvesting energy for further use, which makes the information receivers to may be eavesdrop or intercept during the communication durations. Thus, there are two problems in this system that need to be solved, which are energy sharing during edge nodes and power control for the cognitive network. We first assume that all the I PUs are friendly nodes [29-31] and all the channels are characterized by quasistatic fading models. Then, system models are performed in the next sections.

2.1. Channel Model. The signals received by $\mathrm{SU}_{k}, k \in\{1,2$, $\cdots, K\}$, and $\mathrm{ER}_{m}, m \in\{1,2, \cdots, M\}$, denoted by $y_{k}^{\mathrm{SU}}$ and $y_{m}^{\mathrm{ER}}$ , respectively, can be given as

$$
\begin{gathered}
y_{k}^{\mathrm{SU}}=\mathbf{h}_{k}^{H} \mathbf{x}+n_{k}^{\mathrm{SU}}, \\
y_{m}^{\mathrm{ER}}=\mathbf{g}_{m}^{H} \mathbf{x}+n_{m}^{\mathrm{ER}},
\end{gathered}
$$

where $h_{k}^{H} \in \mathbb{C}^{N_{t} \times 1}$ and $g_{m}^{H} \in \mathbb{C}^{N_{t} \times 1}$ are the channel vectors between the CBS and the $k$ th SU and the $m$ th ER, respectively. In (1), $x \in \mathbb{C}^{N_{t} \times 1}$ is the signal vector transmitted by the CBS to SUs and ERs. $n_{k}^{\mathrm{SU}}$ and $n_{m}^{\mathrm{ER}}$ are the complex Gaussian noise at SUs and ERs with zero mean and variances $\sigma_{\mathrm{SU}_{k}}^{2}$ and $\sigma_{\mathrm{ER}_{m}}^{2}$, which include terminal noise, signal processing noise, interference from PBS, and joint effects of received multicell interference.

In addition, the $\mathrm{PU}_{i}, i \in\{1,2, \cdots, I\}$, receiver is interfered by $\mathrm{CBS}$, and the interference signal is

$$
y_{i}^{\mathrm{PU}}=\mathbf{p}_{i}^{H} \mathbf{x}
$$

where $p_{i}^{H} \in \mathbb{C}^{N_{t} \times 1}$ is channel vector of the CBS to the $i$ th PU receiver. The interference from the PBS to the secondary network is negligible as other researches [30-32].

2.2. CSI. Based on channel reciprocities [7, 33, and 34], it is assumed that $\mathbf{h}_{k}^{H}, \forall k \in\{1,2, \cdots, K\}$, is accusable for both CBS and SUs. However, channel vectors $\mathbf{g}_{m}^{H}$ and $\mathbf{p}_{i}^{H}$ may not be known accurately, as the PUs, CBS, and ERs have no cooperation existing. As a result, we introduce probabilistic
CSI models [21, 33, and 35] to characterize the outdated channel vector errors. The channel vectors follow stochastic distributions, which are

$$
\begin{gathered}
\mathbf{g}_{m}=\widehat{\mathbf{g}}_{m}+\Delta \mathbf{g}_{m}, \\
\Delta \mathbf{g}_{m} \sim \mathscr{C} \mathcal{N}\left(0, \mathbf{G}_{m}\right), \\
m \in\{1,2, \cdots, M\} ; \\
\mathbf{q}_{i}=\widehat{\mathbf{q}}_{i}+\Delta \mathbf{q}_{i}, \\
\Delta \mathbf{q}_{i} \sim \mathscr{C} \mathcal{N}\left(0, \mathbf{Q}_{i}\right), \\
i \in\{1,2, \cdots, I\},
\end{gathered}
$$

where $\mathbf{g}_{m}$ and $\mathbf{q}_{i}$ are the channel estimated CSI available at the CBS and $\Delta \mathbf{g}_{m}$ and $\Delta \mathbf{q}_{i}$ denote the channel error vectors, respectively, with zero mean and covariance matrices $\mathbf{G}_{m}$ and $\mathbf{Q}_{i}$, correspondingly. In addition, $\Delta \mathbf{g}_{m}$ is independent of others for different $m$, and $\Delta \mathbf{q}_{i}$ is independent of others for different $i$.

2.3. Signal and Secure Rate Models. Actually, the ERs in the cognitive network may be illegal eavesdroppers as they have shorter transmit distance from the CBS. Thus, the secrecy of secondary users should be considered. In this paper, we propose a robust $\mathrm{AN}$-aided communication strategy for CBS to efficiently transmit wireless information to end users. The transmitted signal vector $x \in \mathbb{C}^{N_{t} \times 1}$ is added with an artificial noise vector, which is

$$
\mathbf{x}=\sum_{k=1}^{K} \mathbf{w}_{k} d_{k}+\mathbf{v},
$$

where $w_{k} \in \mathbb{C}^{N_{t} \times 1}$ is the dedicated beamforming vector allocated to $\mathrm{SU}_{k}$ and $d_{k} \in \mathbb{C}$ denotes the data symbol for $\mathrm{SU}_{k}$. Without loss of generality, we assumed that $\varepsilon\left\{\left|d_{k}\right|^{2}\right\}=1, \forall k$ $\in\{1,2, \cdots, K\} . v \in \mathbb{C}^{N_{t} \times 1}$ is the $\mathrm{AN}$ vector transmitted by the CBS with $\mathbf{v} \sim \mathscr{C} \mathscr{N}(0, \mathbf{V})$, in which $\mathbf{V}$ is the covariance matrix of $\mathrm{AN}$ and $\mathbf{V} \in H^{N_{t}}, \mathbf{V} \geq 0$. Thus, the achievable data rate $(\mathrm{ADR})$ of the transmission link between the CBS and $\mathrm{S}$ $\mathrm{U}_{k}$ is 


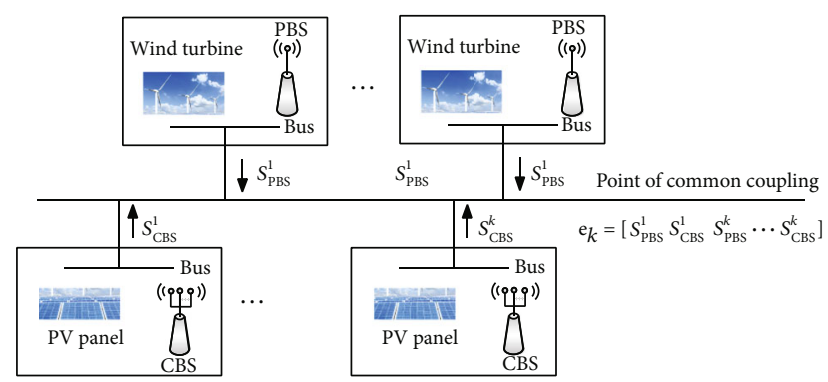

FIGURE 2: System power supply model.

$$
C_{\mathrm{SU}_{k}}=\log _{2}\left(1+\mathrm{SINR}_{\mathrm{SU}_{k}}\right)
$$

and the signal to interference plus noise ratio (SINR) $\mathrm{SINR}_{\mathrm{SU}_{k}}$ can be denoted as

$$
\operatorname{SINR}_{\mathrm{SU}_{k}}=\frac{\left|\mathbf{h}_{k}^{H} \mathbf{w}_{k}\right|^{2}}{\operatorname{Tr}\left(\mathbf{V h}_{k} \mathbf{h}_{k}^{H}\right)+\sigma_{\mathrm{SU}_{k}}^{2}} .
$$

On the other hand, the ADR $C_{\mathrm{ER}_{m}}$ between the CBS and $\mathrm{ER}_{i}$ and SINR of $\mathrm{ER}_{m}$ are given by

$$
\begin{gathered}
C_{\mathrm{ER}_{m}}=\log _{2}\left(1+\mathrm{SINR}_{\mathrm{ER}_{m}}\right), \\
\mathrm{SINR}_{\mathrm{ER}_{m}}=\frac{\left|\mathbf{g}_{m}^{H} \mathbf{w}_{k}\right|^{2}}{\operatorname{Tr}\left(\mathbf{V} \mathbf{g}_{m} \mathbf{g}_{m}^{H}\right)+\sigma_{\mathrm{ER}_{m}}^{2}} .
\end{gathered}
$$

Since the ERs are potential eavesdroppers, the secrecy rate of $\mathrm{SU}_{k}$ is $R_{k}=\min _{m \in\{1,2, \cdots, M\}}\left[C_{\mathrm{SU}_{k}}-C_{\mathrm{ER}_{m}}\right]^{+}$.

2.4. System Power Supply Model. As previously described, a dedicated edge power grid is built to solve the energy sharing problem. CBSs and PBSs are selected to form a MEC network with energy sharing abilities. As shown in Figure 2, wind turbines and photovoltaic panels are appropriately prepared for MEC nodes as green energy harvesting devices. Furthermore, redundant energy of those MEC nodes can be shared with each other through the power grid. Theoretically, the SWIPT-enabled CR MEC framework with energy sharing can be easily expended for unlimited scale and infinite computing abilities. Here, we denote that the energy shared by the PBS and CBS is $S_{\mathrm{PBS}}$ and $S_{\mathrm{CBS}}$, respectively. Then, the power loss of the grid caused by lines is given by

$$
P_{L}=\mathbf{e}_{s} \mathbf{B e}_{s}{ }^{T}, \quad \mathbf{e}_{s}=\left[S_{\mathrm{PBS}}^{1} S_{\mathrm{CBS}}^{1} \cdots S_{\mathrm{PBS}}^{k} S_{\mathrm{CBS}}^{k}\right]
$$

where $B \in \mathbb{R}^{2 \times 2}, \mathbf{B}>0$, is a $B$-coefficient matrix [34] which represents the energy consumption characters during its conversion process. It is noted that, for a fixed power grid topology with fixed numbers of sources and loads, the $B$ -coefficient matrix is constant.

\section{Problem Formulation}

The energy balance can be realized by the sharing abilities of the MEC node based on the CR framework. In particular, the secure AN-aided SWIPT beamforming strategy is designed in each single CR network to minimize the MEC node transmit power under constraints of secrecy rate outage probability, energy harvesting, interferences, etc.:

$$
\begin{gathered}
P 1: \min _{V, e_{s}, w_{k}} \sum_{k=1}^{K}\left\|w_{k}\right\|_{2}^{2}+\operatorname{Tr}(V) \\
\text { s.t. } C_{1}: \operatorname{Pr}\left\{\left[C_{S U_{k}}-C_{E R_{m}}\right]^{+} \geq R_{\min }\right\} \geq 1-\theta, \\
C_{2}: \operatorname{Pr}\left\{\mu\left(\operatorname{Tr}\left(V g_{m} g_{m}^{H}\right)+\sum_{k=1}^{K}\left|g_{m}^{H} w_{k}\right|^{2}+\sigma_{E R}^{2}\right) \geq \Omega_{E R_{m}}\right\} \geq 1-\theta_{E R_{m}}, \\
C_{3}: \operatorname{Pr}\left\{\sum_{k=1}^{K} q_{i}^{H}\left(w_{k} w_{k}^{H}\right) q_{i} \leq P_{\max }\right\} \geq 1-\theta_{P}, \\
C_{4}: P_{P B S}+P_{C B S}+\rho\left(\sum_{k=1}^{K}\left\|w_{k}\right\|_{2}^{2}+\operatorname{Tr}(V)\right) \leq e_{s} 1-e_{s} B e_{s}^{T}, \\
C_{5}: \operatorname{Tr}(V)+\sum_{k=1}^{K}\left\|w_{k}\right\|_{2}^{2} \leq P_{C B S}^{\max }, \\
C_{6}: 0 \leq e_{s} \leq e_{s}^{\max }, \\
C_{7}: V \geq 0,
\end{gathered}
$$

where $R_{\min }$ denotes the CBS minimum secrecy requirement; $\theta \in(0,1], \theta_{\mathrm{ER}_{m}} \in(0,1]$, and $\theta_{P} \in(0,1]$ are the maximum outage probabilities of secrecy rate, the maximum tolerable interference power at PUs, and the minimum energy harvesting requirement of $k$ th $\mathrm{ER}$, respectively; $\Omega_{\mathrm{ER}_{m}}$ denotes the minimum required power transfer to $\operatorname{ER}_{m}$; and $\mu \in(0,1]$ represents the RF-to-DC conversion efficiency of the ERs. $P_{\mathrm{CBS}}^{\max }$ in constraint $C_{4}$ indicates the allowance max-power for transmission at the CBS. In $C_{5}, P_{\mathrm{PBS}}$ and $P_{\mathrm{CBS}}$ represent the localized power expenditure of the PBS and the CBS; the term $\sum_{k=1}^{K}\left\|\mathbf{w}_{k}\right\|^{2}+\operatorname{Tr}(\mathbf{V})$ is the output power of CBS, and $\rho \in(0,1]$ denotes the power efficiency of the power amplifier. The maximum power transmitting to the power grid from energy sources is constrained by $C_{6}$.

\section{Robust Secure SWIPT Beamforming Design}

$\mathrm{P} 1$ is an optimization problem with nonconvex forms and probabilistic constraints, which make P1 difficult to solve. Approximations based on Bernstein-type inequalities are first facilitated to transfer the outage probabilistic constraints $C_{1}, C_{2}$, and $C_{3}$ into clear and closed forms separately and safely. Then, a 1-D algorithm is proposed for computers to calculate the optimal solutions.

Lemma 1 (Bernstein-type inequalities $[34,35])$. Let $f(z)=$ $\mathbf{z}^{H} \mathbf{A z}+2 \operatorname{Re}\left\{\mathbf{z}^{H} \mathbf{b}\right\}+c$, where $\mathbf{A} \in H^{N_{t}}, \mathbf{b} \in \mathbf{C}^{N_{t} \times 1}$, and $c \in \mathbb{R}$ . For any $\omega \in(0,1]$, the approximate and convex forms transferred by $\operatorname{Pr}\{f(z) \geq 0\} \geq 1-\omega$ and $\operatorname{Pr}\{f(z) \leq 0\} \geq 1-\omega$ can be separately written by 


$$
\begin{gathered}
\operatorname{Tr}(\mathbf{A})-\sqrt{-2 \ln (\Phi)} v_{1}+\ln (\Phi) v_{2}+c \geq 0, \\
\left\|\left[\begin{array}{c}
v e c(\mathbf{A}) \\
\sqrt{2} \mathbf{b}
\end{array}\right]\right\| \leq v_{1}, \\
v_{2} \mathbf{I}+\mathbf{A} \geq 0, \\
v_{2} \geq 0 ; \\
\operatorname{Tr}(\mathbf{A})+\sqrt{-2 \ln (\omega)} v_{1}-\ln (\omega) v_{2}+c \leq 0, \\
\left\|\left[\begin{array}{c}
v e c(\mathbf{A}) \\
\sqrt{2} \mathbf{b}
\end{array}\right]\right\| \leq v_{1}, \\
v_{2} \mathbf{I}-\mathbf{A} \geq 0, \\
v_{2} \geq 0,
\end{gathered}
$$

where $v_{1}$ and $v_{2}$ are slack variables.

$$
\begin{aligned}
& \text { Let } \mathbf{W}_{k}=\mathbf{w}_{k} \mathbf{w}_{k}^{H}, \\
& \Psi=\frac{1}{1+\left(\left|\mathbf{h}_{k}^{H} \mathbf{w}_{k}\right|^{2} / \operatorname{Tr}\left(\mathbf{V h}_{k} \mathbf{h}_{k}^{H}\right)+\sigma_{\mathrm{SU}_{k}}^{2}\right)}=\frac{\mathbf{h}_{k}^{H} \mathbf{V} \mathbf{h}_{k}+\sigma_{\mathrm{SU}_{k}}^{2}}{\mathbf{h}_{k}^{H}(\mathbf{V}+\mathbf{W}) \mathbf{h}_{k}+\sigma_{\mathrm{SU}_{k}}^{2}},
\end{aligned}
$$

$\Delta \mathbf{g}_{m}=\mathbf{G}_{m}^{1 / 2} \widetilde{\mathbf{g}}_{m}, \tilde{\mathbf{g}}_{m} \sim \mathscr{C} \mathscr{N}(0, \mathbf{I})$. By applying Lemma 1, $C_{1}$ can be transferred to

$$
\begin{aligned}
& \operatorname{Tr}\left(\mathbf{G}_{m}^{1 / 2}\left[\mathbf{V}-2^{R_{\min }} \Psi\left(\mathbf{V}+\mathbf{W}_{k}\right)\right] \mathbf{G}_{m}^{1 / 2}\right)-\sqrt{-2 \ln \theta} v_{s 1}+\ln \theta v_{s 2} \\
& +\widehat{\mathbf{g}}_{m}^{H}\left[\mathbf{V}-2^{R_{\min }} \Psi\left(\mathbf{V}+\mathbf{W}_{k}\right)\right] \widehat{\mathbf{g}}_{m}+\sigma_{\mathrm{ER}_{m}}^{2}\left(1-2^{R_{\min }} \Psi\right) \geq 0,
\end{aligned}
$$

$$
\begin{gathered}
\left\|\left[\begin{array}{c}
\left.\operatorname{vec}\left(\mathbf{G}_{m}^{1 / 2}\left[\mathbf{V}-2^{R_{\min }} \Psi\left(\mathbf{V}+\mathbf{W}_{k}\right)\right] \mathbf{G}_{m}^{1 / 2}\right)\right] \\
\sqrt{2} \mathbf{G}_{m}^{1 / 2}\left[\mathbf{V}-2^{R_{\min }} \Psi\left(\mathbf{V}+\mathbf{W}_{k}\right)\right] \widehat{\mathbf{g}}_{m}
\end{array}\right]\right\| \leq v_{s 1}, \\
v_{s 2} \mathbf{I}+\mathbf{G}_{m}^{1 / 2}\left[\mathbf{V}-2^{R_{\min }} \Psi\left(\mathbf{V}+\mathbf{W}_{k}\right)\right] \mathbf{G}_{m}^{1 / 2} \geq 0 \\
v_{s 2} \geq 0
\end{gathered}
$$

where $v_{s 1}$ and $v_{s 2}$ are slack variables.

Note. In the equation $f(z)=\mathbf{z}^{H} \mathbf{A z}+2 \operatorname{Re}\left\{\mathbf{z}^{H} \mathbf{b}\right\}+c$, the conversion process from $C_{1}$ to (13) is realized by

$$
\begin{gathered}
\mathbf{z} \text { equivalents to } \tilde{\mathbf{g}}_{m}, \\
\text { A equivalents to } \mathbf{G}_{m}^{1 / 2}\left[\mathbf{V}-2^{R_{\min }} \Psi\left(\mathbf{V}+\mathbf{W}_{k}\right)\right] \mathbf{G}_{m}^{1 / 2}, \\
\text { b equivalents to } \mathbf{G}_{m}^{1 / 2}\left[\mathbf{V}-2^{R_{\min }} \Psi\left(\mathbf{V}+\mathbf{W}_{k}\right)\right] \widehat{\mathbf{g}}_{m}, \\
\text { c equivalents to } \widehat{\mathbf{g}}_{m}^{H}\left[\mathbf{V}-2^{R_{\min }} \Psi\left(\mathbf{V}+\mathbf{W}_{k}\right)\right] \widehat{\mathbf{g}}_{m}+\sigma_{\mathrm{ER}_{m}}^{2}\left(1-2^{R_{\min }} \Psi\right) .
\end{gathered}
$$

Proof. (see the appendix).
Similar to the constraint $C_{1}$, based on Lemma $1, C_{2}$ can be approximated as

$$
\begin{gathered}
\operatorname{Tr}\left(\mathbf{G}_{m}^{1 / 2}\left(\mathbf{V}+\mathbf{W}_{k}\right) \mathbf{G}_{m}^{1 / 2}\right)-\sqrt{-2 \ln \theta_{\mathrm{ER}_{m}}} v_{e 1}+\ln \theta_{\mathrm{ER}_{m}} v_{e 2} \\
+\widehat{\mathbf{g}}_{m}^{H}\left(\mathbf{V}+\mathbf{W}_{k}\right) \widehat{\mathbf{g}}_{m}+\sigma_{\mathrm{ER}_{m}}^{2}-\frac{\Omega_{\mathrm{ER}_{m}}}{\mu} \geq 0 \\
\left\|\left[\begin{array}{c}
\operatorname{vec}\left(\mathbf{G}_{m}^{1 / 2}\left(\mathbf{V}+\mathbf{W}_{k}\right) \mathbf{G}_{m}^{1 / 2}\right) \\
\sqrt{2} \mathbf{G}_{m}^{1 / 2}\left(\mathbf{V}+\mathbf{W}_{k}\right) \widehat{\mathbf{g}}_{m}
\end{array}\right]\right\| \leq v_{e 1} \\
v_{e 2} \mathbf{I}+\mathbf{G}_{m}^{1 / 2}\left(\mathbf{V}+\mathbf{W}_{k}\right) \mathbf{G}_{m}^{1 / 2} \geq 0
\end{gathered}
$$

where $v_{e 1}$ and $v_{e 2}$ are slack variables. Let $\Delta \mathbf{q}_{i}=\mathbf{Q}_{i}^{1 / 2} \tilde{\mathbf{q}}_{i}, \tilde{\mathbf{q}}_{i} \sim$ $\mathscr{C} \mathscr{N}(0, \mathbf{I})$, using Lemma 1 , constraint $C_{3}$ is approximated by

$$
\begin{gathered}
\operatorname{Tr}\left(\mathbf{Q}_{i}^{1 / 2}\left(\mathbf{V}+\mathbf{W}_{k}\right) \mathbf{Q}_{i}^{1 / 2}\right)+\sqrt{-2 \ln \theta_{P}} v_{p 1}-\ln \theta_{P} v_{p 2} \\
+\widehat{\mathbf{q}}_{i}^{H}\left(\mathbf{V}+\mathbf{W}_{k}\right) \widehat{\mathbf{q}}_{i}-P_{\max } \geq 0, \\
\left\|\left[\begin{array}{c}
\operatorname{vec}\left(\mathbf{Q}_{i}^{1 / 2}\left(\mathbf{V}+\mathbf{W}_{k}\right) \mathbf{Q}_{i}^{1 / 2}\right) \\
\sqrt{2} \mathbf{Q}_{i}^{1 / 2}\left(\mathbf{V}+\mathbf{W}_{k}\right) \widehat{\mathbf{q}}_{i}
\end{array}\right]\right\| \leq v_{p 1}, \\
v_{p 2} \mathbf{I}-\mathbf{Q}_{i}^{1 / 2}\left(\mathbf{V}+\mathbf{W}_{k}\right) \mathbf{Q}_{i}^{1 / 2} \succeq 0,
\end{gathered}
$$

where $v_{p 1}$ and $v_{p 2}$ are slack variables.

Although the approximated forms of (13), (18), and (21) are deterministic forms, the problem P1 is still difficult to solve due to the nonconvex caused by fractional and coupling constraints. In particular, we take advantage of the characteristics of fractional parameter $\Psi$, which can be seen that $0<$ $\Psi \leq 1$. Thus, for a given $\Psi$, suboptimal computed solutions can be obtained by a proposed 1-D search method. The relaxation of $\mathrm{P} 1$ is

$$
\begin{array}{cc}
\text { P2 : } & \min _{\mathbf{V}, \mathbf{e}_{s}, \mathbf{W}_{k},\left\{v_{s 1}\right\},\left\{v_{s 2}\right\},\left\{v_{e 1}\right\},\left\{v_{e 2}\right\},\left\{v_{p 1}\right\},\left\{v_{p 2}\right\}} \sum_{k=1}^{K} \operatorname{Tr}\left(\mathbf{W}_{k}\right)+\operatorname{Tr}(\mathbf{V}) \\
\text { s.t. } & \mathbf{h}_{k}^{H}\left((\Psi-1) \mathbf{V}+\Psi \mathbf{W}_{k}\right) \mathbf{h}_{k}+(\Psi-1) \sigma_{\mathrm{SU}_{k}}^{2}=0 \\
(1),(2),(3), C 4-C 7 \\
C_{8}: R\left(\mathbf{W}_{k}\right) \leq 1, \mathbf{W}_{k} \geq 0, \mathbf{W}_{k} \in \mathbb{H}^{N_{t}}
\end{array}
$$

in which $C_{8}$ guarantees that $\mathbf{W}_{k}=\mathbf{w}_{k} \mathbf{w}_{k}^{H}$. As $C_{8}$ is a rank-one, a safe relaxation is introduced to make $\mathrm{P} 2$ convex by removing $C_{8}$ from P2's constraints. Then, the optimal solutions are obtained by convex programming software CVX [28].

Algorithm 1 is the detail of the 1-D algorithm. In this case, parameters $\theta, \theta_{\mathrm{ER}_{m}}$, and $\theta_{P}$ have to be initialized and the search interval of the uniform search variable $\Psi$ is $(0,1]$. It is noted that the problem P2 cannot make the matrices $\mathbf{W}_{k}$ rank-one. If the solutions $\mathbf{W}_{k}$ are rank-one for all SUs, the optimal secure beamforming vector can be obtained through eigenvalue decomposing of $\mathbf{W}_{k}$. If the solutions $\mathbf{W}_{k}$ 
1: Initialization: $R_{\min }, \theta, \mu, \Omega_{E R_{m}}, \theta_{E R_{m}}, P_{\max }, \theta_{P}, P_{C B S}^{\max }, P_{P B S}, P_{C B S}, \rho$.

2: Input: $\mathbf{h}_{k}^{H}, k=1,2, \cdots, K, \widehat{\mathbf{g}}_{m}, m=1,2, \cdots, M, \widehat{\mathbf{q}}_{i}, i=1,2, \cdots, I$.

3: Set: The iteration index $n=1$, the step length factor of the

1-D search $\tau$.

\section{4: Optimization:}

5: for $\Psi=\tau: \tau: 1$

6: solve the $\mathrm{P} 3$,

$$
\begin{aligned}
\mathrm{P} 3: & \min _{\mathbf{V}, \mathbf{e}_{s}, \mathbf{w}_{k},\left\{v_{s 1}\right\},\left\{v_{s 2}\right\},\left\{v_{e 1}\right\},\left\{v_{e 2}\right\},\left\{v_{p 1}\right\},\left\{v_{p 2}\right\}} \sum_{k=1}^{K} \operatorname{Tr}\left(\mathbf{W}_{k}\right)+\operatorname{Tr}(\mathbf{V}) \\
& \text { s.t. } \mathbf{h}_{k}^{H}\left((\Psi-1) \mathbf{V}+\Psi \mathbf{W}_{k}\right) \mathbf{h}_{k}+(\Psi-1) \sigma_{S U_{k}}^{2}=0
\end{aligned}
$$

$$
\text { (1), (2), (3), C4-C7 }
$$

obtain $\left\{\mathbf{W}_{1}, \mathbf{W}_{2}, \cdots, \mathbf{W}_{K}\right\}^{n}, \mathbf{V}^{n}, \mathbf{e}_{s}{ }^{n},\left(\sum_{k=1}^{K} \operatorname{Tr}\left(\mathbf{W}_{k}\right)+\operatorname{Tr}(\mathbf{V})\right)^{n}$;

set $n=n+1$.

7: end

8: Comparison: Find the optimal $\left\{\mathbf{W}_{1}, \mathbf{W}_{2}, \cdots, \mathbf{W}_{K}\right\}^{\text {opt }}, \mathbf{V}^{\text {opt }}, \mathbf{e}_{s}^{\text {opt }},\left(\sum_{k=1}^{K} \operatorname{Tr}\left(\mathbf{W}_{k}\right)+\operatorname{Tr}(\mathbf{V})\right)^{o p t}$ from the $n\left\{\mathbf{W}_{1}, \mathbf{W}_{2}, \cdots, \mathbf{W}_{K}\right\}^{n}, \mathbf{V}^{n}, \mathbf{e}_{s}^{n}$, $\left(\sum_{k=1}^{K} \operatorname{Tr}\left(\mathbf{W}_{k}\right)+\operatorname{Tr}(\mathbf{V})\right)^{n}$

Algorithm 1: The 1-D search algorithm.

TABLE 1: Parameters for simulation.

\begin{tabular}{lc}
\hline Parameters & Values \\
\hline Minimum secrecy rate requirement & $R_{\min }=1.5 \mathrm{bits} / \mathrm{s} / \mathrm{Hz}$ \\
Energy conversion efficiency & $\mu=0.9$ \\
Minimum required power of $\mathrm{ER}_{m}$ & $\Omega_{\mathrm{ER}_{m}}=5 \mathrm{~W}$ \\
Maximum tolerable interference at PU & $P_{\text {max }}=5 \mathrm{~W}$ \\
Outage probabilities & $\theta=0.05, \theta_{\mathrm{ER}_{m}}=0.05, \theta_{P}=0.05$ \\
Maximum transmit power allowance at the CBS & $P_{\mathrm{CBS}}^{\max }=10 \mathrm{~W}$ \\
Fixed power expenditure & $P_{\mathrm{PBS}}=50 \mathrm{~W}, P_{\mathrm{CBS}}=50 \mathrm{~W}$ \\
Channel power gains & $\mathbf{h}_{k} \sim \mathscr{C} \cdot \mathcal{N}(0, \mathbf{I}), \widehat{\mathbf{g}}_{m} \sim C N(0, \mathbf{I}), \widehat{\mathbf{q}}_{i} \sim C N(0, \mathbf{I})$ \\
Variances of noise & $\sigma_{\mathrm{SU}_{k}}^{2}=\sigma_{\mathrm{ER}_{m}}^{2}=0.05$
\end{tabular}

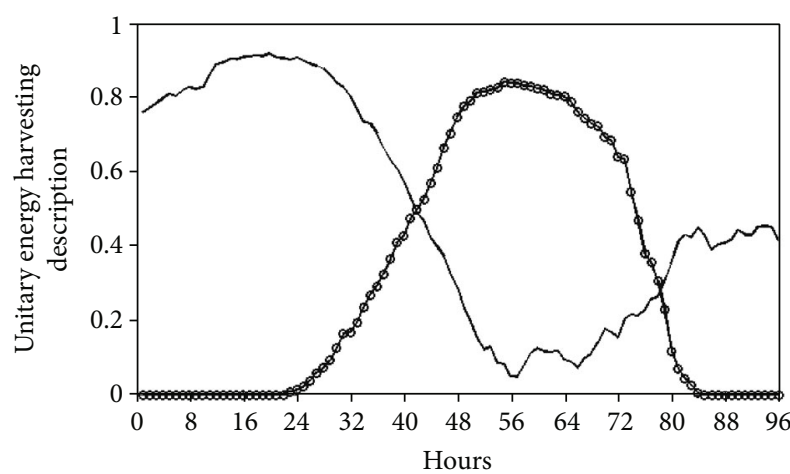

- Wind energy

$\rightarrow$ Solar energy

Figure 3: System power supply model.

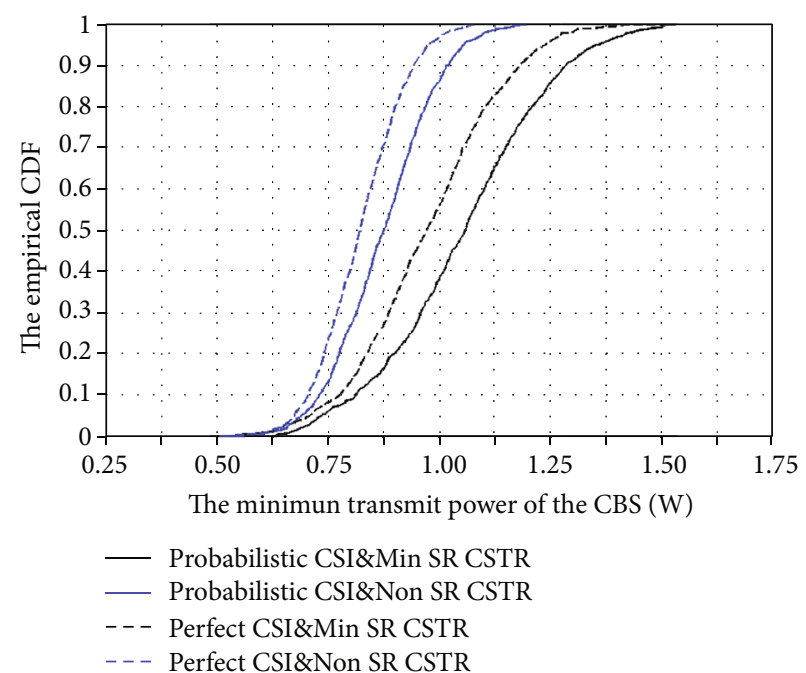

FIgURE 4: The CDF of the minimum power for transmission of the CBS. 


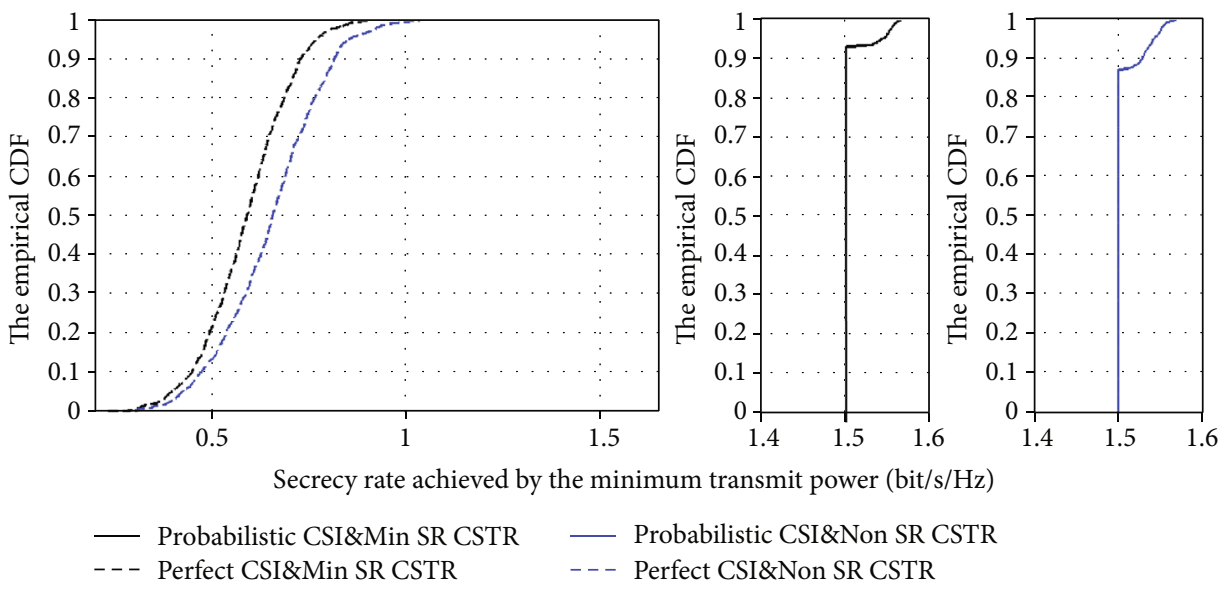

FIGURE 5: The CDF of the secrecy rate achieved by the minimum transmit power of the CBS.

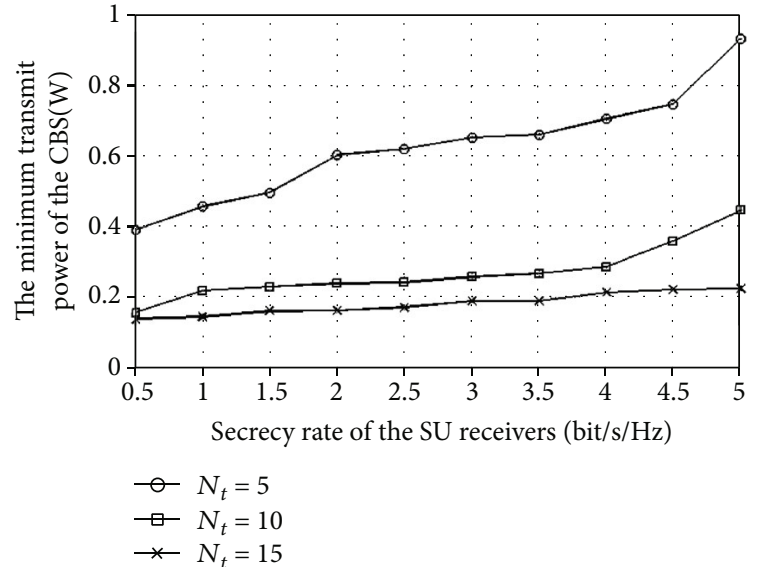

(a)

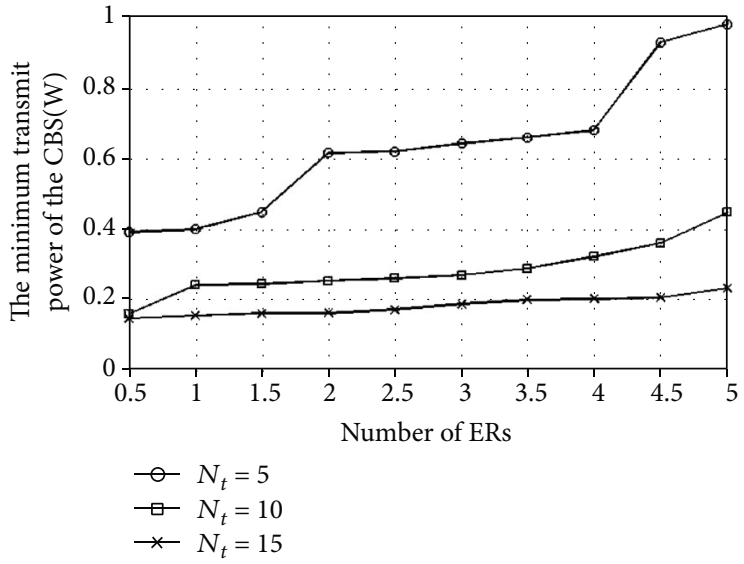

(b)

FIGURE 6: The CBS minimum transmit power under different secrecy rate requirements and different numbers of ERs. (a) The relationship between the CBS minimum transmit power and the number of ERs. (b) The relationship between the CBS minimum transmit power and the secrecy rate requirements.

are not rank-one, Gaussian randomization procedure can be used to find the suboptimal solutions. Meanwhile, the corresponding optimal objective value provides the lower bound of (24) [35].

\section{Simulation Results}

Based on the proposed 1-D algorithm, this section illustrates the system gains by simulations. The SWIPT-enabled CR MEC network is considered with 2 SUs, 3 ERs, and 4 PUs. The antennas of the CBS are $N_{t}=5$. Table 1 details the parameters for simulations. We discuss the system gains realized by the secure beamforming compared with ideal cases.

The real energy harvesting data are descripted in Figure 3, which are collected by Guangxi Power Grid Co., Ltd with the intervals of fifteen minutes. In practice, the information transmission coherence times are small enough, which can be ignored compared with the power transfer intervals. Thus, $\mathbf{e}_{s}^{\max }$ is supposed to be a known constant; that is to say, the PBS and CBS can use energy harvested in current intervals based on the knowledge of harvesting status. Those approaches contribute to the constraint $C_{5}$ in problem formulations; the energy cooperation is realized between PBS and CSB until the problem is solved and the average minimum energy sequences are obtained.

Figure 4 is 500 calculations for the CDF of the CBS optimized transmit power under the probabilistic CSI with minimum secrecy rate (SR) constraint, the probabilistic CSI without minimum SR constraint, the perfect CSI with minimum SR constraint, and the perfect CSI without minimum SR constraint, separately. It can be seen that the minimum power transmitted by the CBS under the probabilistic CSI error models is bigger than the other scenes as the channel uncertainty takes more energy consumptions to meet the expected secrecy rate. Figure 5 indicates the CDF of the secrecy rate of the 500 calculations, from which we can see that with the minimum secrecy rate constraint, the system can achieve higher security rates and the imperfect CSI significantly reduce the system preference. It is worth noting that the cumulative probabilities under probabilistic CSI and 
perfect CSI scenes are started from the position of the lowest safety rate, and the minimum secrecy rate is $1.5 \mathrm{bit} / \mathrm{s} / \mathrm{Hz}$. The vertical parts of the CDF curves are caused by the introduction of secrecy rate constraints.

The minimum power transmitted by CBS is shown in Figure 6 under different secrecy rate requirements and different numbers of ERs. $N_{t}$ is set as $N_{t}=5, N_{t}=10$, or $N_{t}=15$. Figure 6(a) concluded that a higher secure rate results in greater energy consumption. Furthermore, the transmit power of CBS decreases with $N_{t}$ increases; this is because more antennas degrees the source allocation with higher degrees of freedom. Figure 6(b) describes the relationship between ERs and CBS optimal transmit power.

\section{Conclusions}

This paper proposes a SWIPT-enabled CR framework for MEC networks. A dedicated edge power grid is built for sharing energy among MEC nodes to balance the energy distribution. In particular, robust $\mathrm{AN}$-aided secure beamforming is designed for CR networks to realize secure communications and optimal power control. Bernstein-type inequalities are introduced to transfer the problem into closed and safely relaxed forms. The solutions are obtained with the designed 1-D algorithm. Simulation results proved the effectiveness and performance of the proposed scheme. System gains are also achieved with the cost of implementation complexities.

\section{Appendix}

\section{The Proof of Lemma 1}

Let $\mathbf{W}_{k}=\mathbf{w}_{k} \mathbf{w}_{k}^{H}$ and $\Psi=\left(\mathbf{h}_{k}^{H} \mathbf{V h} \mathbf{h}_{k}+\sigma_{\mathrm{SU}_{k}}^{2}\right) /\left(\mathbf{h}_{k}^{H}\left(\mathbf{V}+\mathbf{W}_{k}\right) \mathbf{h}_{k}+\right.$ $\left.\sigma_{\mathrm{SU}_{k}}^{2}\right)$, we can rewrite the inequality $\left[C_{\mathrm{SU}_{k}}-C_{\mathrm{ER}_{m}}\right]^{+} \geq R_{\min }$ as the following form:

$$
\frac{\mathbf{g}_{m}^{H} \mathbf{V} \mathbf{g}_{m}+\sigma_{\mathrm{ER}_{m}}^{2}}{\Psi\left(\mathbf{g}_{m}^{H}\left(\mathbf{V}+\mathbf{W}_{k}\right) \mathbf{g}_{m}+\sigma_{\mathrm{ER}_{m}}^{2}\right)}-2^{R_{\min }} \geq 0 .
$$

Due to the nonnegative of the denominator in the above inequality, we have

$$
\mathbf{g}_{m}^{H} \mathbf{V} \mathbf{g}_{m}+\sigma_{\mathrm{ER}_{m}}^{2}-2^{R_{\min }}\left(\Psi\left(\mathbf{g}_{m}^{H}\left(\mathbf{V}+\mathbf{W}_{k}\right) \mathbf{g}_{m}+\sigma_{\mathrm{ER}_{m}}^{2}\right)\right) \geq 0
$$

After the equation $\mathbf{g}_{m}=\widehat{\mathbf{g}}_{m}+\Delta \mathbf{g}_{m}, \Delta \mathbf{g}_{m} \sim \mathscr{C} \mathcal{N}\left(0, \mathbf{G}_{m}\right)$, $m \in\{1,2, \cdots, M\}$, is substituted and performed some algebraic manipulations; (A.2) can be rewritten as

$$
\begin{aligned}
\Delta \mathbf{g}_{m}^{H} & {\left[\mathbf{V}-2^{R_{\min }} \Psi\left(\mathbf{V}+\mathbf{W}_{k}\right)\right] \Delta \mathbf{g}_{m}+\Delta \mathbf{g}_{m}^{H}\left[\mathbf{V}-2^{R_{\min }} \Psi\left(\mathbf{V}+\mathbf{W}_{k}\right)\right] \widehat{\mathbf{g}}_{m} } \\
& +\widehat{\mathbf{g}}_{m}^{H}\left[\mathbf{V}-2^{R_{\min }} \Psi\left(\mathbf{V}+\mathbf{W}_{k}\right)\right] \Delta \mathbf{g}_{m}+\widehat{\mathbf{g}}_{m}^{H}\left[\mathbf{V}-2^{R_{\min }} \Psi\left(\mathbf{V}+\mathbf{W}_{k}\right)\right] \widehat{\mathbf{g}}_{m} \\
& +\sigma_{\mathrm{ER}_{m}}^{2}\left(1-2^{R_{\min }} \Psi\right) \geq 0 .
\end{aligned}
$$

Let $\Delta \mathbf{g}_{m}=\mathbf{G}_{m}^{1 / 2} \tilde{\mathbf{g}}_{m}, \tilde{\mathbf{g}}_{m} \sim \mathscr{C} \mathscr{N}(0, \mathbf{I})$, then the first term of the polynomial of the left side in the inequality (A.3) is rewritten as

$$
\begin{aligned}
& \Delta \mathbf{g}_{m}^{H}\left[\mathbf{V}-2^{R_{\min }} \Psi\left(\mathbf{V}+\mathbf{W}_{k}\right)\right] \Delta \mathbf{g}_{m} \\
& =\tilde{\mathbf{g}}_{m}{ }^{H} \mathbf{G}_{m}^{1 / 2^{H}}\left[\mathbf{V}-2^{R_{\min }} \Psi\left(\mathbf{V}+\mathbf{W}_{k}\right)\right] \mathbf{G}_{m}^{1 / 2} \tilde{\mathbf{g}}_{m} .
\end{aligned}
$$

Based on the expression $f(z)=\mathbf{z}^{H} \mathbf{A} \mathbf{z}+2 \operatorname{Re}\left\{\mathbf{z}^{H} \mathbf{b}\right\}+c$ in Lemma $1, \mathbf{A}$ is equivalent to $\mathbf{G}_{m}^{1 / 2 H}\left[\mathbf{V}-2^{R_{\min }} \Psi\left(\mathbf{V}+\mathbf{W}_{k}\right)\right] \mathbf{G}_{m}^{1 / 2}$ and $\mathbf{z}$ is equivalent to $\tilde{\mathbf{g}}_{m}$. Then, the rest of polynomial is converted as

$$
\begin{aligned}
& \Delta \mathbf{g}_{m}^{H}\left[\mathbf{V}-2^{R_{\min }} \Psi\left(\mathbf{V}+\mathbf{W}_{k}\right)\right] \widehat{\mathbf{g}}_{m}+\widehat{\mathbf{g}}_{m}^{H}\left[\mathbf{V}-2^{R_{\min }} \Psi\left(\mathbf{V}+\mathbf{W}_{k}\right)\right] \Delta \mathbf{g}_{m} \\
& =\tilde{\mathbf{g}}_{m}{ }^{H} \mathbf{G}_{m}^{1 / 2 H}\left[\mathbf{V}-2^{R_{\min }} \Psi\left(\mathbf{V}+\mathbf{W}_{k}\right)\right] \widehat{\mathbf{g}}_{m}+\widehat{\mathbf{g}}_{m}^{H}\left[\mathbf{V}-2^{R_{\min }} \Psi\right. \\
& \left.\left(\mathbf{V}+\mathbf{W}_{k}\right)\right] \mathbf{G}_{m}^{1 / 2} \tilde{\mathbf{g}}_{m}=\tilde{\mathbf{g}}_{m}{ }^{H} \mathbf{G}_{m}^{1 / 2} \quad\left[\mathbf{V}-2^{R_{\min }} \Psi\left(\mathbf{V}+\mathbf{W}_{k}\right)\right] \widehat{\mathbf{g}}_{m} \\
& +\widehat{\mathbf{g}}_{m}^{H}\left[\mathbf{V}-2^{R_{\min }} \Psi\left(\mathbf{V}+\mathbf{W}_{k}\right)\right] \mathbf{G}_{m}^{1 / 2} \tilde{\mathbf{g}}_{m} \\
& =2 \operatorname{Re}\left\{\tilde{\mathbf{g}}_{m}{ }^{H} \cdot \mathbf{G}_{m}^{1 / 2}\left[\mathbf{V}-2^{R_{\min }} \Psi\left(\mathbf{V}+\mathbf{W}_{k}\right)\right] \widehat{\mathbf{g}}_{m}\right\} \text {. }
\end{aligned}
$$

Therefore, $\mathbf{b}$ in the expression $f(z)=\mathbf{z}^{H} \mathbf{A z}+2 \operatorname{Re}\left\{\mathbf{z}^{H} \mathbf{b}\right\}$ $+c$ is equivalent to $\mathbf{G}_{m}^{1 / 2}\left[\mathbf{V}-2^{R_{\min }} \Psi\left(\mathbf{V}+\mathbf{W}_{k}\right)\right] \widehat{\mathbf{g}}_{m}$, and $c$ is equivalent to $\hat{g}_{m}^{H}\left[V-2^{R_{\min }} \Psi\left(V+W_{k}\right)\right] \widehat{g}_{m}+\sigma_{E R_{m}}^{2}\left(1-2^{R_{\min }} \Psi\right)$.

Finally, the constraint $C_{1}$ can be equivalently expressed as (A.6), where $v_{e 1}$ and $v_{e 2}$ are slack variables:

$$
\begin{aligned}
\Psi= & \frac{\mathbf{h}_{k}^{H} \mathbf{V h}_{k}+\sigma_{\mathrm{SU}_{k}}^{2}}{\mathbf{h}_{k}^{H}\left(\mathbf{V}+\mathbf{W}_{k}\right) \mathbf{h}_{k}+\sigma_{\mathrm{SU}_{k}}^{2}} \operatorname{Tr}\left(\mathbf{G}_{m}^{1 / 2}\left[\mathbf{V}-2^{R_{\min }} \Psi\left(\mathbf{V}+\mathbf{W}_{k}\right)\right] \mathbf{G}_{m}^{1 / 2}\right) \\
& -\sqrt{-2 \ln \theta} v_{s 1}+\ln \theta v_{s 2}+\widehat{\mathbf{g}}_{m}^{H}\left[\mathbf{V}-2^{R_{\min }} \Psi\left(\mathbf{V}+\mathbf{W}_{k}\right)\right] \widehat{\mathbf{g}}_{m} \\
& +\sigma_{\mathrm{ER}_{m}}^{2}\left(1-2^{R_{\min }} \Psi\right) \geq 0,
\end{aligned}
$$

$$
\begin{gathered}
\left\|\left[\begin{array}{c}
\left.\operatorname{vec}\left(\mathbf{G}_{m}^{1 / 2}\left[\mathbf{V}-2^{R_{\min }} \Psi\left(\mathbf{V}+\mathbf{W}_{k}\right)\right] \mathbf{G}_{m}^{1 / 2}\right)\right] \\
\sqrt{2} \mathbf{G}_{m}^{1 / 2}\left[\mathbf{V}-2^{R_{\min }} \Psi\left(\mathbf{V}+\mathbf{W}_{k}\right)\right] \widehat{\mathbf{g}}_{m}
\end{array}\right]\right\| \leq v_{s 1}, \\
v_{s 2} \mathbf{I}-\mathbf{G}_{m}^{1 / 2}\left[\mathbf{V}-2^{R_{\min }} \Psi\left(\mathbf{V}+\mathbf{W}_{k}\right)\right] \mathbf{G}_{m}^{1 / 2} \geq 0 \\
v_{s 2} \geq 0
\end{gathered}
$$

This completes the proof.

\section{Data Availability}

This paper is based on mathematical models of wireless networks including the channel model, communication model, energy model, and computing models.

\section{Conflicts of Interest}

The authors declare that there is no conflict of interest regarding the publication of this paper. 


\section{Acknowledgments}

This work was supported in part by the National Natural Science Foundation of China under Grant 61762010, in part by the Guangxi University for Nationalities Introduction of Talents Research Project Foundation under Grant 2019KJQD17, in part by the Natural Science Foundation of Guangxi under Grant 2018GXNSFAA294155, and also in part by the Beijing Intelligent Logistics System Collaborative Innovation Center under Grant BILSCIC-2019KF-07.

\section{References}

[1] C.-H. Hsu, S. Wang, Y. Zhang, and A. Kobusinska, "Mobile edge computing," Wireless Communications and Mobile Computing, vol. 2018, Article ID 7291954, 3 pages, 2018.

[2] J. Zhang, W. Xie, F. Yang, and Q. Bi, "Mobile edge computing and field trial results for 5G low latency scenario," China Communications, vol. 13, no. 2, pp. 174-182, 2016.

[3] R. L. Varshney, "Transporting information and energy simultaneously," in 2008 IEEE International Symposium on Information Theory, Toronto, ON, Canada, July 2008.

[4] R. Zhang and C. K. Ho, "MIMO broadcasting for simultaneous wireless information and power transfer," IEEE Transactions on Wireless Communications, vol. 12, no. 5, pp. 1989-2001, 2013.

[5] K. Xiong, C. Chen, G. Qu, P. Fan, and K. B. Letaief, "Group cooperation with optimal resource allocation in wireless powered communication networks," IEEE Transactions on Wireless Communications, vol. 16, no. 6, pp. 3840-3853, 2017.

[6] K. Xiong, B. Wang, and K. J. R. Liu, "Rate-energy region of SWIPT for MIMO broadcasting under nonlinear energy harvesting model," IEEE Transactions on Wireless Communications, vol. 16, no. 8, pp. 5147-5161, 2017.

[7] F. Zhou, N. C. Beaulieu, J. Cheng, Z. Chu, and Y. Wang, "Robust max-min fairness resource allocation in sensingbased wideband cognitive radio with SWIPT: imperfect channel sensing," IEEE Systems Journal, vol. 12, no. 3, pp. 23612372, 2018.

[8] R. Liu and W. Trappe, In Securing Wireless Communications at the Physical Layer, Springer-Verlag, New York, NY, USA, 1st edition, 2010.

[9] Y. Pei, Y.-c. Liang, L. Zhang, K. Teh, and K. Li, "Secure communication over MISO cognitive radio channels," IEEE Transactions on Wireless Communications, vol. 9, no. 4, pp. 14941502, 2010.

[10] D. W. K. Ng, E. S. Lo, and R. Schober, "Robust beamforming for secure communication in systems with wireless information and power transfer," IEEE Transactions on Wireless Communications, vol. 13, no. 8, pp. 4599-4615, 2014.

[11] Y. Lu, K. Xiong, P. Fan, Z. Ding, Z. Zhong, and K. B. Letaief, "Global energy efficiency in secure MISO SWIPT systems with non-linear power-splitting EH model," IEEE Journal on Selected Areas in Communications, vol. 37, no. 1, pp. 216232, 2019.

[12] J. Liu, Y. Gu, L. Zha, Y. Liu, and J. Cao, "Event-Triggered $H_{\infty}$ Load Frequency Control for Multiarea Power Systems Under Hybrid Cyber Attacks," IEEE Transactions on Systems, Man, and Cybernetics: Systems, vol. 49, no. 8, pp. 1665-1678, 2019.

[13] L. Zha, E. Tian, X. Xie, Z. Gu, and J. Cao, "Decentralized eventtriggered $H_{\infty}$ control for neural networks subject to cyber- attacks," Information Sciences, vol. 457-458, no. 457 , pp. 141-155, 2018.

[14] D. W. K. Ng and R. Schober, "Max-min in fair wireless energy transfer for secure multiuser communication systems," in IEEE Information Theory Workshop (ITW 2014), pp. 326330, Hobart, TAS, Australia, 2014.

[15] R. Feng, Q. Li, Q. Zhang, and J. Qin, "Robust Secure Transmission in MISO Simultaneous Wireless Information and Power Transfer System," IEEE Transactions on Vehicular Technology, vol. 64, no. 1, pp. 400-405, 2015.

[16] J. Guang and A. L. Swindlehurst, "Robust secure transmission in MISO channels based on worst-case optimization," IEEE Transactions on Signal Processing, vol. 60, pp. 1696-1702, 2012.

[17] W. Wu, X. J. Yin, P. Deng, T. W. Guo, and B. Y. Wang, “Transceiver Design for Downlink SWIPT NOMA Systems With Cooperative Full-Duplex Relaying," IEEE Access, vol. 7, pp. 33464-33472, 2019.

[18] W. Wu, F. H. Zhou, P. Li, P. Deng, B. Y. Wang, and V. C. M. Leung, "Energy-efficient resource allocation for secure NOMA-enabled mobile edge computing networks," IEEE Transactions on Communications, vol. 68, no. 1, pp. 493-505, 2020.

[19] Q. Li and W. K. Ma, "Spatially selective artificial-noise aided transmit optimization for MISO multi-eves secrecy rate maximization," IEEE Transactions on Signal Processing, vol. 61, no. 10 , pp. 2704-2717, 2013.

[20] W. C. Liao, T. H. Chang, W. K. Ma, and C. Chi, "QoS-based transmit beamforming in the presence of eavesdroppers: an optimized artificial noise-aided approach," IEEE Transactions on Signal Processing, vol. 59, no. 3, pp. 1202-1216, 2011.

[21] D. W. K. Ng, E. S. Lo, and R. Schober, "Energy-efficient resource allocation for secure OFDMA systems," IEEE Transactions on Vehicular Technology, vol. 61, no. 6, pp. 2572-2585, 2012.

[22] Y. Lu, K. Xiong, P. Fan, Z. Zhong, and K. B. Letaief, "Robust transmit beamforming with artificial redundant signals for secure SWIPT system under non-linear EH model," IEEE Transactions on Wireless Communications, vol. 17, no. 4, pp. 2218-2232, 2018.

[23] F. Zhou, Z. Li, J. Cheng, Q. Li, and J. Si, "Robust AN-aided beamforming and power splitting design for secure MISO cognitive radio with SWIPT," IEEE Transactions on Wireless Communications, vol. 16, no. 4, pp. 2450-2464, 2017.

[24] M. Grant and S. Boyd, "CVX: Matlab software for disciplined convex programming, version 2.0 beta," 2013, http-s://http:// cvxr.com/cvx.

[25] X. Lu, P. Wang, D. Niyato, D. I. Kim, and Z. Han, "Wireless networks with RF energy harvesting: a contemporary survey," IEEE Communications Surveys \& Tutorials, vol. 17, no. 2, pp. 757-789, 2015.

[26] R. K. Sharma and D. B. Rawat, "Advances on security threats and countermeasures for cognitive radio networks: a survey," IEEE Communications Surveys \& Tutorials, vol. 17, no. 2, pp. 1023-1043, 2015.

[27] N. Mokari, S. Parsaeefard, H. Saeedi, P. Azmi, and E. Hossain, "Secure robust ergodic uplink resource allocation in relayassisted cognitive radio networks," IEEE Transactions on Signal Processing, vol. 63, no. 2, pp. 291-304, 2015.

[28] B. Fang, Z. Qian, W. Zhang, and W. Shao, "An-aided secrecy precoding for SWIPT in cognitive MIMO broadcast channels," 
IEEE Communications Letters, vol. 19, no. 9, pp. 1632-1635, 2015.

[29] Z. Chu, H. Xing, M. Johnston, and S. Le Goff, "Secrecy Rate Optimizations for a MISO Secrecy Channel with Multiple Multiantenna Eavesdroppers," IEEE Transactions on Wireless Communications, vol. 15, no. 1, pp. 283-297, 2016.

[30] Z. Chu, Z. Zhu, M. Johnston, and S. Y. Le Goff, "Simultaneous wireless information power transfer for MISO secrecy channel," IEEE Transactions on Vehicular Technology, vol. 65, no. 9, pp. 6913-6925, 2016

[31] S. Ma and D. Sun, "Chance constrained robust beamforming in cognitive radio networks," IEEE Communications Letters, vol. 17, no. 1, pp. 67-70, 2013.

[32] A. J. Wood and B. F. Wollenberg, "Power generation operation and control - 2nd edition," Fuel and Energy Abstracts, vol. 37, no. 3, 1996.

[33] K.-Y. Wang, A. M.-C. So, T.-H. Chang, W.-K. Ma, and C.Y. Chi, "Outage constrained robust transmit optimization for multiuser MISO downlinks: tractable approximations by conic optimization," IEEE Transactions on Signal Processing, vol. 62, no. 21, pp. 5690-5705, 2014.

[34] I. Bechar, "A Bernstein-type inequality for stochastic processes of quadratic forms of Gaussian variables," Mathematics, 2009, http://arxiv.org/abs/ 0909.3595v1.

[35] Z. Luo, W. Ma, A. M. So, Y. Ye, and S. Zhang, "Semidefinite relaxation of quadratic optimization problems," IEEE Signal Processing Magazine, vol. 27, no. 3, pp. 20-34, 2010. 